\title{
Courses
}

- Joachim Cuntz (Münster)

Title: Cyclic homology.

Abstract: We give an introduction to basic ideas and concepts in cyclic homology.

- Bernhard Keller (Paris 7)

Title: $D g$ categories.

Abstract: Derived categories were invented by Grothendieck-Verdier in the early sixties in order to formulate Grothendieck's duality theory for schemes. Today, they have become an important tool in many branches of algebraic geometry, in algebraic analysis, non commutative algebraic geometry, representation theory, mathematical physics ... . In an attempt to axiomatize the properties of derived categories, Grothendieck-Verdier introduced the notion of a triangulated category. For a long time, triangulated categories were considered too poor to allow the development of more than a rudimentary theory. This vision has changed in recent years (notably thanks to Neeman's and Balmer's work), but the fact remains that many important constructions of derived categories cannot be performed with triangulated categories. Notably, tensor products and functor categories formed from triangulated categories are no longer triangulated. One approach to overcome these problems has been the theory of derivators initiated by Heller and Grothendieck at the beginning of the nineties. Another, perhaps less formidable one is the theory of differential graded categories ( $=d g$ categories), together with its cousin, the theory of $A$ - $\infty$-categories. In this survey, we review the foundations of dg categories and report on recent work by Drinfeld, Dugger-Shipley, ... Toën and Toën-Vaquié.

- Boris Tsygan (Northwestern)

Title: Characteristic classes in noncommutative geometry.

Abstract: I will outline several constructions of characteristic classes. I will start with the Connes-Karoubi Chern character from K theory to negative cyclic homology. Then I will generalize it to obtain the Chern character of perfect complexes of sheaves of modules over a sheaf of rings. I will also recall Karoubi's generalized regulators, and their generalization to perfect complexes. I will end by an application of these constructions to generalized Chern-Simons classes of D-modules.

- Charles Weibel (Rutgers)

Title: Motivic cohomology.

Abstract: This is the program for the three lectures.

Lecture 1: We introduce the notion of sheaves and presheaves with transfers, define Motivic Cohomology, and explain the relation to Milnor $K$-theory. The "Tate motives" $\mathbb{Z}(i)[n]$ are chain complexes of sheaves with transfers.

Lecture 2: The triangulated category $D M$ of motives is defined, and shown to contain the classical category of Chow Motives, due to Grothendieck, as well as the category of geometric motives, both of which have good duality theories. In this context, the Motivic Cohomology of $X$ becomes the bigraded groups $\operatorname{Hom}(X, \mathbb{Z}(i)[n])$.

Lecture 3: Cohomology operations can be defined on $D M$, and lead to a proof of the Milnor Conjecture. Similar ideas form the basis for the announced proof of the so-called Bloch-Kato Conjecture by Voevodsky and Rost. A sketch of some key ideas involved in the proof will be given. 


\section{Invited Talks}

- Paul Balmer (Zürich)

Title: Triangular Geometry

Abstract: Triangulated categories appear in many areas of mathematics and quite crucially around K-theory. Recently they also entered the theory of $C^{*}$-algebras. In this talk, I'll explain how to study abstract tensor triangulated categories via geometric ideas.

- Paul Baum (Penn State)

Title: Non-commutative geometry and the representation theory of $p$-adic groups.

Abstract:Non-commutative geometry begins with the Gelfand theorem asserting that commutative $C^{*}$-algebras and locally compact Hausdorff topological spaces are the same thing. Another classical theorem states that unital commutative finitely-generated nilpotentfree algebras (over the complex numbers) is the same thing as complex affine algebraic varieties. This can be taken as the starting point for non-commutative algebraic geometry. Based on this point of view, the talk states a conjecture within the representation theory of $p$-adic groups. The idea of the conjecture is that a simple geometric structure underlies many delicate and intricate results in this representation theory.

The above is joint work with Anne-Marie Aubert and Roger Plymen.

- Stefan Gille (München)

Title: A Gersten-Witt complex for Azumaya algebras with involution.

Abstract: Let $X$ be a regular noetherian scheme of finite Krull dimension, e.g. a smooth scheme over a field, and $\mathcal{A}$ an Azumaya algebra over $X$ with involution $\tau$ of the first kind. If $1 / 2$ is in the global sections of $X$ then there exists a spectral sequence which converges to the derived hermitian Witt theory of $(\mathcal{A}, \tau)$. It turns out that the odd lines of this spectral are zero, and the even lines $2 q$ are isomorphic to Gersten-Witt complexes:

$$
\begin{aligned}
\bigoplus_{x \in X^{(0)}} W^{(-1)^{q}}(\mathcal{A}(x), \tau(x)) \stackrel{d^{0}}{\longrightarrow} & \bigoplus_{x \in X^{(1)}} W^{(-1)^{q}}(\mathcal{A}(x), \tau(x)) \stackrel{d^{1}}{\longrightarrow} \ldots \\
\ldots & \longrightarrow \bigoplus_{x \in X^{(i)}} W^{(-1)^{q}}(\mathcal{A}(x), \tau(x)) \stackrel{d^{i}}{\longrightarrow} \ldots
\end{aligned}
$$

Here $X^{(p)} \subseteq X$ denotes the set of points of codimension $p, k(x)$ the residue field of $x \in$ $X, \mathcal{A}(x)$ is the central simplex $k(x)$-algebra $\mathcal{A} \otimes k(x)$ with induced involution $\tau(x)$, and $W^{ \pm 1}(\mathcal{A}(x), \tau(x))$ denotes the (skew-)hermitian Witt group of this central simple $k(x)$ algebra with involution.

These complexes are exact if $X$ is the spectrum of a local ring of a smooth variety and the kernel of $d^{0}$ is then naturally isomorphic to $W^{+1}(\mathcal{A}, \tau)$ in the hermitian case, i.e. for $q$ even, and to $W^{-1}(\mathcal{A}, \tau)$ in the skew-hermitian case, i.e. for $q$ odd.

Such a spectral sequence exists also if the involution is of the second kind. The odd lines of this spectral sequence are then also zero, however the even lines look differently. In this case nothing is known about the Gersten-conjecture, i.e. the exactness of this complex for $X$ the spectrum of a local ring of a smooth variety.

- Victor Ginzburg (University of Chicago)

Title: Calabi-Yau algebras.

Abstract: We introduce a new class of associative algebras called Calabi-Yau algebras. Calabi-Yau algebras arise in Mirror symmetry; the category of coherent sheaves on a Calabi-Yau variety is quite often equivalent to the category of modules over an appropriate Calabi-Yau algebra. 
Calabi-Yau algebras are defined in terms of a noncommutative 'potential', and such algebras enjoy a number of nice homological properties. We consider a few concrete examples of Calabi-Yau algebras associated with quivers, with McKay quivers, in particular.

- Alexander Gorokhovsky (Boulder)

Title: Deformation quantization of étale groupoids and gerbes.

Abstract: This is a joint work with P.Bressler, R. Nest and B. Tsygan. We will discuss problems in which formal deformations of etale groupoids and gerbes arise and give an explicit description of the differential graded Lie algebra which controls this deformation theory.

- Maxim Kontsevich (IHES)

Title: On the degeneration of the Hodge to de Rham spectral sequence.

Abstract: Several years ago I proposed a conjecture saying that for a smooth proper $\mathrm{dg}$ algebra over a field of characteristic zero, the spectral sequence from Hochschild homology to the periodic cyclic homology collapses. In the case of the dg algebra describing perfect complexes over a smooth proper scheme (Bondal - van der Bergh theorem) this is just the usual classical degeneration of the Hodge to de Rham spectral sequence. Recently D.Kaledin claimed a proof of my conjecture in the special case of a sheaf of algebras on a site, generalizing Deligne-Illusie method. In my talk I wille describe 3 conjectures related the degeneration conjecture, weaking some assumptions on dg algebras.

- Marc Levine (Northeastern)

Title: Algebraic cobordism.

Abstract: We will give a report on some recent work on algebraic cobordism, joint with R. Pandharipande. Algebraic cobordism was defined by myself and Fabien Morel as a geometric construction of the "classical" part of $M G L$-theory. We have also shown that algebraic cobordism is the universal oriented cohomology theory on smooth varieties, and have related algebraic cobordism to the Lazard ring via its formal group law. This theory has been useful in proving the degree formulas of Markus Rost, as well as giving a systematic understanding of Riemann-Roch theorems.

Together with Pandharipande, we have given a simple presentation of the algebraic cobordism groups of a variety via "double point cobordisms". We use this to verify some conjectures of Pandharipande et al. on generating functions arising in Donaldson-Thomas theory.

- Wolfgang Lück (Münster)

Title: The Farrell-Jones Conjecture for algebraic $K$-theory holds for word-hyperbolic groups and arbitrary coefficients.

Abstract: This is joint work with Arthurs Bartels and Holger Reich from Muenster. The main result is that the Farrell-Jones Conjecture for algebraic K-theory is true for wordhyperbolic groups and arbitrary cefficient rings. This result has many application, e.g., it implies the Bass Conjecture for fields of characteristic zero and Moody's induction theorem for word hyperbolic groups. We show that the Farrell-Jones Conjecture for algebraic $K$ theory is true for all coefficient rings for those groups which were defined by Gromov and for which the Baum-Connes Conjecture with coefficients fails as shown by Higson, Lafforgue and Skandalis. The methods of proof are based on $K$-theory, geometric group theory and controlled topology. 
- Ralf Meyer (Göttingen)

Title: Bivariant K-theory: where topology and analysis meet.

Abstract: In noncommutative topology, we study ill-behaved moduli spaces by attaching $C *$-algebras to them. The advantage over other approaches to moduli spaces like stacks is that the homological study of $C *$-algebras is rather simple: in a precise sense, any reasonable homology theory for $C *$-algebras is closely related to $K$-theory.

This can be formalised using the bivariant $K$-theory of Kasparov, which is a substitute for the stable homotopy category and carries similar structure. In addition, bivariant $K$ theory brings some functional analysis into play, which we can use to solve problems that cannot be solved by purely topological means.

An important instance of this is the question whether or not the Baum-Connes assembly map is an isomorphism. Whereas the construction of this assembly map is a purely topological affair, proofs of injectivity or bijectivity depend on analysis, which in turn often leads to notions in geometric group theory.

- Amnon Neeman (Canberra)

Title: The homotopy category of flat modules, and Grothendieck's local duality.

Abstract: Let $X$ be a scheme, and let $D^{b}(C o h / X)$ be the derived category of bounded complexes of coherent sheaves on $X$. It may happen that this category is equivalent to its dual. This phenomenon was first studied by Grothendieck, as part of his duality theory. A "dualizing complex" on $X$ is enough to guarantee that the category $D^{b}(\mathrm{Coh} / X)$ is self-dual.

This part of Grothendieck's theory, known as "local duality", is perhaps the least well understood. In the 40 years since Grothendieck's duality theorem we have come to understand certain aspects of it quite well, but there really hasn't been a drastically different, more illuminating approach to local duality. We basically do not understand, even after 40 years, why the category $D^{b}(C o h / X)$ is so often self-dual.

In the last couple of years there have been a few articles, by Krause, Jorgensen, and Iyengar and Krause, which have thrown a totally new light on the problem. We will discuss their results, as well as related work by the speaker.

- Ryszard Nest (Copenhagen)

Title: Applications of triangulated structure of KK-theory to quantum groups.

Abstract: In this talk we will show how to formulate Baum Connes conjecture for compact quantum groups and sketch its proof in the case of coaction of compact groups.

- Andreas Thom (Göttingen)

Title:Comparison between algebraic and topological K-theory.

(Joint with Guillermo Cortiñas).

Abstract:This talk is concerned with the algebraic $K$-theory of locally convex algebras stabilized by operator ideals, and its comparison with topological $K$-theory. We show that the obstruction for the comparison map between algebraic and topological $K$-theory to be an isomorphism is (absolute) algebraic cyclic homology and prove the existence of an 6 -term exact sequence. We show that cyclic homology vanishes in the case when $J$ is the ideal of compact operators and $L$ is a Fréchet algebra with bounded app. unit. This proves the generalized version of Karoubi's conjecture due to Mariusz Wodzicki and announced in his paper "Algebraic $K$-theory and functional analysis", First European Congress of Mathematics, Vol. II (Paris, 1992), 485-496, Progr. Math., 120, Birkhuser, Basel, 1994. 
- Mariusz Wodzicki (Berkeley)

Title: Megatrace.

Abstract: All known reasonable constructions of exotic operator traces are specializations of "megatrace".

- Christian Voigt (Münster)

Title:The Baum-Connes conjecture for the dual of $S U_{q}(2)$.

Abstract: We describe a proof of the Baum-Connes conjecture for the dual of the quantum $S U(2)$ group of Woronowicz. The formulation of the conjecture is based on the framework of equivariant KK-theory and its structure as a triangulated category. The proof involves the study of certain equivariant Fredholm modules as well as the equivariant Chern character, Hopf-Galois theory and the representation theory of quantum $S U(2)$. As a byproduct one obtains an interpretation of the Weyl group of the quantum $S U(2)$ group on the level of equivariant $K K$-theory. Finally we explain how to calculate the $K$-groups of $C\left(S U_{q}(2)\right)$ using the validity of the conjecture. 


\section{Communications}

- Marian Anton (University of Kentucky)

Title: Homological symbols.

Abstract: We are concerned with the $\bmod p$ cohomology of the general linear group $G L(n, R)$ of invertible matrices of rank $n$ over a ring of algebraic integers $R$. More specifically, after inverting the prime $p$ we study the image of this cohomology on diagonal matrices. If we conjecture that this image is a certain ring of invariants we discover that some homological classes will vanish in $S L(2, R)$ and vice versa. We call these classes homological symbols by analogy with algebraic $K$-theory symbols and show how to relate them with the arithmetic of the ring $R$ and the structure of the group $S L(2, R)$. Also we calculate an example where these symbols vanish.

- Grzegorz Banaszak (Posnan)

Title: An imbedding property of $K$-groups

Abstract: For a pair of rings $A \subset B$ satisfying certain condition ( $\star$ ) we get general imbedding results for Karoubi-Villamayor, Homotopy and Quillen $K$-theories. One important case is when $A$ and $B$ are respectively the henselization and completion of a regular ring of finite type over a field at a prime ideal.

- Ulrich Bunke (Göttingen)

Title: Duality of topological abelian groups stacks and $T$-duality.

Abstract:

In this talk I want to present results in the following directions:

(1) extension of Pontrjagin duality of locally compact abelian groups to locally compact topological group stacks

(2) interpretation of topological $T$-duality in this context

(3) sheaf theory for topological stacks and application to twisted cohomology and $K$ theory again with emphasis of the context of topological $T$-duality.

- Leandro Cagliero (FAMAF, Córdoba)

Title: The cohomology ring of truncated quiver algebras.

Abstract: In this talk we present a joint work with G. Ames and P. Tirao (see math.KT/0603056) in which we explicitly describe the Yoneda product in the Hochschild cohomology ring $H^{*}(A, A)$ of an arbitrary truncated quiver algebra $A$ in terms of a minimal resolution of A.

It is known that the Yoneda product has a nice description on the bar resolution of $A$ as the usual cup product. On the other hand, the cohomology groups has been described by Locateli in terms of classes of pairs of paths using minimal resolutions.

Our first result is the explicit construction of comparison morphisms, in both directions, between the two different resolutions. We are then able to give a clear description of the Yoneda product on the minimal resolution that, in particular, implies that the product of two cohomology classes of odd degree is equal to zero. Our main theorem extends, to arbitrary truncated quiver algebras, the results obtained by Bardzell, Locateli and Marco for cyclic truncated quiver algebras. In addition, we show that the ring structure (of the augmentation ideal) is trivial if the underlying quiver is not an oriented cycle and has neither sinks nor sources. 
- Paulo Carrillo (Institut Jussieu)

Title: An analytical index for Lie groupoids.

Abstract:

Let $G$ be a Lie groupoid. Using the tangent groupoid associated to it, we propose (in the spirit of Connes-SkandalisMonthubert-Pierrot and Nistor,Weinstein-Xu) an analytical index morphism who takes values in a group where it is possible to apply Chern-Weil theory (Pairings with cyclic cocycles, Haefliger cocycles, etc.). We recall that in general it is not possible to do so for the $K$-theory group of the reduced $C^{*}$-algebra associated to the groupoid.

- Guillermo Cortiñas (Valladolid)

Title: $K$-theory and homological obstructions to regularity.

Abstract: We consider algebras and schemes essentially of finite type over a field $F$ of characteristic zero. In a joint paper with Haesemeyer, Schlichting and Weibel (math.KT/0502255), we computed the fiber $\mathcal{F}_{K}(X)$ of the map $K(X) \rightarrow K H(X)$ going from $K$-theory to homotopy $K$-theory in terms of cyclic homology. We showed $\mathcal{F}_{K}(X)$ coincides, up to a delooping, with the fiber $\mathcal{F}_{H C}(X)$ of the canonical map going from $H C(X)$ to its cdh-fibrant replacement $\mathbb{H}_{c d h}(X, H C)$. This computation proved rather useful to attack problems concerning how $K$-theory detects singularity. For example we showed in loc. cit. that if $\operatorname{dim} X=d$ then $K_{n}(X)=0$ for $n<d$ and $X$ is $K_{-d}$-regular, thus establishing in characteristic zero a conjecture of Weibel. For another example we proved in our joint paper with Haesemeyer and Weibel (math.KT/042606), that if an algebra $R$ is of dimension $d$ and $K_{d+1}$-regular then it is regular, thus establishing in characteristic zero an old conjecture of Vorst. In ongoing joint research with Haesemeyer and Weibel we have studied Bass' question: does $K_{n}(R)=K_{n}(R[t])$ imply $K_{n}(R)=K_{n}(R[t, u])$ ? We show that this problem can be equivalently formulated in terms of Hochschild homology, prove some positive partial results, and relate Bass' question to Rodicio's conjecture on vanishing of $H H$ and to Berger's conjecture on the torsion of differentials of an affine curve.

- Mathias Fuchs (Luminy)

Title: Cyclic cohomology associated to higher rank lattices and the idempotents of the group ring.

Abstract: We review topologically Nistor's computation of the homogeneous part of the periodic cyclic cohomology of crossed products $\Gamma \ltimes A$ of torsion-free discrete groups $\Gamma$ with a complex $\Gamma$-algebra $A$. Let $B$ be a minimal parabolic subgroup of $G$. Applied to torsion-free discrete cocompact subgroups $\Gamma$ of complex connected semisimple Lie groups $G$ and the smooth functions on the Furstenberg boundary $G / B$, this yields under a weak topological condition, together with the classical splitting principle, the surjectivity of the map $H P^{*}\left(\Gamma \ltimes \mathcal{C}^{\infty}(G / B)\right) \rightarrow H P^{*}(\mathbb{C} \Gamma)$ on the periodic cyclic cohomology of the map of smooth convolution groupoids associated to $G / B \rightarrow p t$. Furthermore, the algebraic $K$ theory group $K_{0}\left(\Gamma \ltimes \mathcal{C}^{\infty}(G / B)\right)$ can be calculated in analogy to the $C^{*}$-algebraic case by a Dirac-dual Dirac method. As a consequence, the trace functional can be evaluated on the whole of $K_{0}(\mathbb{C} \Gamma)$ and is integer if $G=S L(n, \mathbb{C})$. We thus obtain a new proof of the idempotent conjecture for the group rings of the considered class of groups.

- Imma Gálvez (London Metropolitan University)

Title: Up-to-homotopy structures on Vertex Algebras

Abstract: (joint work with V Gorbounov (Aberdeen) and A Tonks (London))

We give a proof of a conjecture of Lian and Zuckerman (1993), see also Kimura et al (1997), Conj 2.3. We were motivated by interest in the algebraic structures on the chiral De Rham complex, see Gorbounov et al (2004). 
Lian and Zuckerman show that BRST cohomology has a natural Gerstenhaber algebra structure and claim this lifts to a homotopy structure at the chain level. A partial solution was given by Voronov (2000).

This is a frequent phenomenon in the world of operads: an associative algebra structure on the homology of a loop space $Y$ lifts to an $A_{\infty}$-space structure on $Y$; also the Gerstenhaber algebra structure on Hochschild cohomology reflects homotopy algebra structure on the complex of Hochschild cochains.

We present an elementary definition of $G_{\infty}$-algebra, compare Tamarkin-Tsygan (2000), and introduce (partial) $G_{r}$-algebra structures. Inspired by work on strong homotopy Lie algebras by Barnich et al (1998) we give an obstruction for extension of $G_{r^{-}}$to $G_{r+1^{-}}$ structures in terms of the null homotopy of a certain structure map.

- Grigory Garkusha (Manchester)

Title: Triangulated categories of rings.

Abstract: A kind of unstable homotopy theory on the category of associative rings (without unit) is developed. There are the notions of fibrations, homotopy (in the sense of Karoubi), path spaces, Puppe sequences etc. One introduces the notion of a quasiisomorphism (or weak equivalence) for rings and shows that - similar to spaces - the derived category obtained by inverting the quasi-isomorphisms is naturally left triangulated. Then one stabilizes the loop fuctor resulting a triangulated category for rings and constructs a bivariant theory with some character maps. Also, homology theories on rings are studied. These must be homotopy invariant in the algebraic sense, meet the Mayer-Vietoris property and some additional minor natural properties. To any functor $X$ from rings to pointed simplicial sets a homology theory is associated in a natural way. If $X=G L$ one recovers Karoubi-Villamayor functors $K V_{i}, i>0$. If $X$ is Quillen's $K$-theory functor, one recovers the (non-negative) homotopy $K$-theory in the sense of Weibel. Technically, we use the homotopy information for the category of simplicial functors on rings and apply Bousfield localization theory for model categories.

- Juan José Guccione (Buenos Aires)

Title: Relative cyclic homology of square zero extensions.

Abstract: Let $k$ be a characteristic zero field, $C$ a $k$-algebra and $M$ a square zero two sided ideal of $C$. We obtain a new mixed complex, simpler than the canonical one, giving the Hochschild and cyclic homologies of $C$ relative to $M$. This complex resembles the canonical reduced mixed complex of an augmented algebra. We begin the study of our complex showing that it has a harmonic decomposition like to the one considered by Cuntz and Quillen for the normalized mixed complex of an algebra. We also give new proofs of two theorems of Goodwillie, obtaining a light improvement of one of them. Finally, under suitable conditions we have a simpler mixed complex, wich allow us to obtain explicit computations.

- Fernando Muro (MPI, Bonn)

Title: On the 1-type of Waldhausen $K$-theory.

Abstract: This is a report on joint work with Andrew Tonks. We construct a small functorial model for the 1-type of a Waldhausen $K$-theory spectrum. This model consists of a very special kind of symmetric monoidal category called stable quadratic module. The model is defined in terms of generators and relations, and can be regarded as a presentation of $K_{0}, K_{1}$, and the action of the stable Hopf map, all at the same time. We will discuss the multiplicative properties of this model with respect to biexact functors, showing that for monoidal Waldhausen categories our model determines the 1 -type of $K$-theory not only as a spectrum but as a ring spectrum. 
- Wend Werner (Münster)

Title: Ternary rings of operators, affine manifolds and causal structure.

Abstract: On the open unit ball $D$ of a ternary ring of operators $X$ (especially, of a $C^{*}$-algebra) we define an affine connection $\nabla$ which for a number of reasons seems to be the 'natural' one. Its construction is based on a noncommutative hyperbolic structure.

We furthermore consider the problem of characterizing the (local) causal structure on $D$ that comes from embedding $X$ into a space of bounded operators. The embeddings we consider here have to respect both, the canonical connections and the action of the group of biholomorphic mappings. 